\title{
Indicadores socioeconómicos de desarrollo humano y desarrollo relativo al género en la sección municipal de Sabancuy, Carmen, Campeche
}

\author{
Ileana Mercedes Canepa Pérez \\ imcanepa@hotmail.com \\ Fajime Cú Quijano \\ cuquief@hotmail.com
}

Profesoras investigadoras de tiempo completo adscritas a la Facultad de Ciencias Sociales de la Universidad Autónoma de Campeche, México 


\section{RESUMEN}

El objetivo del presente estudio es conocer el grado de desarrollo que se ha alcanzado en la sección municipal de Sabancuy, localizada en el municipio del Carmen, Campeche, México, utilizando la metodología del programa de las Naciones Unidas para el Desarrollo (PNUD) obteniéndose dos indicadores socioeconómicos: El Índice de Desarrollo Humano (IDH) e Índice de Desarrollo Relativo al Género (IDG). El IDH reveló que la sección municipal de Sabancuy tiene un desarrollo humano medio (o.6358) y el IDG fue de 0.6195 , lo que indica que hay una pérdida en los niveles de bienestar cuando se consideran las desigualdades de género.

Palabras clave: Desarrollo, IDH, IDG, bienestar, sección municipal.

\section{Clasificación JEL: O15}

\section{ABSTRACT}

The objective of the present study is to know the level of development that has been reached in the municipal section of Sabancuy, located in the municipality of the Carmen, Campeche, Mexico, using the methodology of the United Nations Development Program (UNDP) and having obtained two socioeconomic indicators: The Human Development Index (HDI) and the Gender related Development Index (GDI). The IDH revealed that the municipal section of Sabancuy has a medium human development (o.6358) and the IDG was of 0.6195 , which indicates that there is a loss in the welfare levels when the inequalities of gender are considered.

Keywords: Development, HDI, GDI, welfare, municipal section.

JEL Clasification: $\mathrm{O} 15$

Revista de Economía, vol. XXVII, núm. 75, segundo semestre de 2010. 


\section{INTRODUCCIÓN}

Generalmente cuando los estudiosos de las Ciencias Sociales investigan sobre el tema de bienestar social hacen referencia a indicadores que midan el avance social y económico de un individuo a partir del PIB per cápita, sin embargo, este indicador tiene varias limitaciones, principalmente por ser una medida promedio, lo cual no permite verificar si toda la población cuenta con acceso al mismo nivel de satisfactores, lo anterior ha llevado a replantear la medición del desarrollo utilizando variables que inciden directamente en la calidad de vida del ser humano, esto último visto como un conjunto amplio de condiciones materiales y el desarrollo de las capacidades básicas como una expansión de las libertades humanas.

Reconocer los valores humanos y de la naturaleza sin duda podrá lograr armonizar las transformaciones de las estructuras productivas con un sentido de equidad distributiva, de ahí la importancia de este enfoque que considera la multiplicidad de la dimensión social, ambiental y cultural del desarrollo (Jiménez, 2001).

El primer estudio sobre desarrollo humano a escala mundial se realizó en 1990 por el Programa de Naciones Unidas para el Desarrollo (PNUD) y a partir de esta fecha estos estudios anuales permiten conocer el grado de bienestar alcanzado por el desarrollo de cada país.

En México los estudios se habían centrado principalmente en medir el bienestar social a partir de la marginación y la pobreza, sin embargo, desde 1995 se realizan trabajos sobre la medición de índices de Desarrollo Humano a nivel estatal y municipal, este último es un ejercicio relativamente reciente en las investigaciones sobre el desarrollo dada las dificultades para la obtención de información estadística, por lo que es necesario sustituir algunas variables y adaptarlas a nivel local.

En el estado de Campeche se carece de trabajos de desarrollo humano que estudien sólo una sección municipal, por ello en la presente investigación se calculan dos indicadores de bienestar social (Índice de Desarrollo Humano e Índice de Desarrollo Relativo al Género) a partir de la 
aplicación de encuestas a una muestra de la población en la sección municipal de Sabancuy en el año 2008, en el municipio del Carmen, Campeche, lo que nos permitió la obtención de los datos más aproximados para la aplicación de la metodología del PNUD.

El interés de esta investigación es proporcionar parámetros que den una idea de los resultados de la acción política y social que se están llevando a cabo para alcanzar el bienestar social en la sección de Sabancuy, asimismo, que les sirva de referencia a los gobiernos locales para diseñar las políticas públicas de acuerdo a las necesidades de la población, con objetivos claros.

La cabecera municipal de la sección de Sabancuy es un viejo asentamiento con raíces coloniales que desde fines del siglo XVIII junto con Chicbul fueron los únicos establecidos - hasta principios del siglo XX-en el área de selva tropical que actualmente constituye su jurisdicción política administrativa. Sus comunidades rurales son de formación relativamente reciente, los 12 ejidos surgieron mediante dos procesos diferenciados pero históricamente entrelazados: a) bajo el impulso del programa federal de colonización agraria de principios de los 1960, donde el gobierno federal redistribuyó en las selvas del sur de Campeche a la población campesina sin tierras de las entidades del norte y centro-occidente del país: Chicbul en 1965, La Cristalina en 1970, Plan de Ayala en 1973 y Abelardo L. Rodríguez en 1978, y b) como resultado de la inmigración espontánea de los 1980 de población rural procedente de las entidades de Veracruz, Chiapas y Tabasco, en esta misma década surge Independencia, Nicolás Bravo, Rodríguez Cano, e Ignacio Gutiérrez; Oxcabal en 1981 y Pino Suárez en 1987 (Dzib, investigación de campo, 2003).

Estos nuevos ejidos y sus poblados han ido desarrollando su economía y vida organizativa dependiendo de las acciones, regulaciones, recursos y programas de la burocracia federal que a nombre de "un estado" benefactor, protector e interventor, controló desde el acceso a la tierra, formación de sus pueblos y la introducción de servicios y obras materiales, hasta el financiamiento, acopio, comercialización y regulación de los precios de sus productos agrícolas (Zendejas, 2003). 
La actuación del gobierno como benefactor generalmente obedece a las condiciones económicas imperantes en cada periodo y con ello la implementación de las políticas públicas, en este sentido el país vive la era de los mercados desregulados, del adelgazamiento de la estructura burocrática del gobierno federal y la desaparición de subsidios al campo, la mayoría de los productores rurales se han visto obligados a realizar trabajos complementarios para subsistir. Los ejidatarios, además de cultivar maíz para su autoconsumo - porque producir para el mercado ya no era rentable- de llevar sus hortalizas y frutas de temporada en pequeña escala a vender en el mercado y las calles de la cabecera de la sección municipal, de recolectar leña y elaborar carbón para los asaderos de pollos, de pescados y de carnes rojas de las ciudades de Sabancuy, Escárcega y del Carmen, se han visto obligados a realizar actividades complementarias como la albañilería, la pesca en las aguadas naturales de Sabancuy y la migración a Estados Unidos (Dzib, investigación de campo 2003).

El declive de las producciones de caña de azúcar y arroz de la era de los subsidios y apoyos gubernamentales de las décadas 1970 y 1980, fue sustituido en el área por el cultivo de chile jalapeño con recursos de los propios ejidatarios en la época de los mercados desregulados de los 1990, la competitividad de los ejidos de la sección municipal en un mercado controlado por los grandes acaparadores del centro del país, no pudo sostenerse cuando aparecieron regiones productoras en distritos de riego con mejor infraestructura productiva. La caída de los precios y de las adquisiciones de los intermediarios, a fines de los 1990, finalmente colapsó la economía de las familias de ejidatarios y no ejidatarios que en las zonas rurales dependían mayoritariamente de actividades agrícolas.

Desde mediados de 1990, la mayoría de dicha población ha tratado de buscar opciones de empleo fuera de la agricultura y del poblado: muchos jóvenes han migrado a Ciudad del Carmen para intentar emplearse en las plataformas (de Pemex) o para acomodarse en los negocios del sector servicios y comercio de la isla que se han multiplicado por influjo del auge petrolero; las jóvenes solteras sin estudios mayormente han empezado a irse de empleadas domésticas a Escárcega, Sabancuy o Ciudad del Carmen. (Dzib, investigación de campo 2003). 
Gran parte de las condiciones socioeconómicas que presenta actualmente la población de la sección municipal de Sabancuy es resultado de las políticas implementadas por el gobierno y del desarrollo de las potencialidades de los individuos para lograr un mejor nivel de vida.

En la sección municipal de Sabancuy también se han creado nuevas oportunidades para el género femenino en el ámbito político, la prueba es que a mediados de noviembre de 2003 destaca el triunfo de una mujer joven de 40 años de edad que ocupa el cargo de agente municipal. Lo relevante de este ascenso femenino era que no constituía un caso aislado en la sección municipal de Sabancuy. Ese año otras mujeres no ejidatarias también ocuparían las agencias municipales de Independencia, Oxcabal y Abelardo Rodríguez. (Dzib U., investigación de campo, 2006), estos acontecimientos muestran que la igualdad de género empieza a entenderse como una oportunidad de desarrollo para mejorar el nivel de vida de las familias, el reducir las desproporciones laborales, salariales y de puestos entre hombres y mujeres coadyuvará a tener una mejor sociedad. Es por ello que cuando se analiza la información de los municipios se debe tomar en cuenta las diferencias de desarrollo entre hombres y mujeres. En este sentido resulta de particular utilidad el índice de desarrollo relativo al género (IDG), porque ajusta el nivel de desarrollo promedio por las desigualdades entre géneros, es decir, señala el nivel de desarrollo humano alcanzado una vez tomadas en cuenta las diferencias entre hombres y mujeres.

\section{MARCO TEÓRICO}

La medición y caracterización de las condiciones de vida de una población son esenciales para el diseño de las políticas públicas de un país. El indicador comúnmente utilizado para medir el nivel de vida de una población ha sido el Producto Interno Bruto (PIB). Sin embargo, este indicador tiene varias limitantes como indicador de bienestar, según lo manifiesta Hernández y Székely (2005). Una de ellas es que el PIB incluye en su cálculo componentes que no necesariamente guardan relación con el bienestar de los hogares y personas. Otra es que al ser un promedio, no permite verificar si toda la población cuenta efectivamente con acceso al mismo nivel de satisfactores. 
$\mathrm{Al}$ respecto, Jiménez (2001) menciona que el desarrollo, entendido casi siempre desde una óptica económica, amplía su visión admitiendo que el progreso humano no es exactamente equivalente al crecimiento económico. La medición de la felicidad, el bienestar y el desarrollo no pueden reflejarse solamente a través de las tasas de incremento de la producción, medidas por el Producto Interno Bruto.

Es de suma importancia incluir el nivel de vida en medidas de índices de desarrollo humano, lo que implica forzosamente hacer mención de la pobreza, como un referente para significar el concepto del nivel de vida.

El concepto de nivel de vida lo abordamos desde el enfoque de las necesidades básicas; según Sen (2001), éstas deberían formularse de acuerdo con las funcionalidades y las posibilidades, en este sentido la forma de necesidades se dan en artículos de consumo, ésta entendida desde su naturaleza derivativa y contingente y de artículos de valor, que son instrumentalmente importantes.

En esta teoría la cuestión principal es la bondad de la vida que una persona puede llevar. Por lo que considera que la necesidad de artículos de consumo para cualquier logro concreto en las condiciones de vida puede variar mucho según las distintas características contingentes, fisiológicas, sociales y culturales, entre otras. Sin embargo, lo interesante es tomar el valor del nivel de vida que reside en la vida misma, y no en la posesión de artículos de consumo, lo cual tiene una importancia derivativa y variable (Sen, 2001).

Pero para alcanzar logros en el nivel de vida que se desea no es cuestión sólo de necesidades básicas, también es necesario saber cómo puede lograrse en una sociedad donde hay instituciones y factores cambiantes del cual dependerá este nivel de vida. La decisión de una persona de lograr lo que desea será determinada por las condiciones de equidad, ésta entendida como la idea fundamental del concepto de justicia.

Rawls (2003) considera la justicia como una virtud de instituciones sociales, o prácticas (cargos, papeles, defensas, etc.). Menciona que por 
ser una virtud de instituciones sociales debe quedar claro que éstas pueden ser anticuadas, ineficientes, degradantes o cualquier otra cosa, sin ser injustas.

Para el estudio de la equidad desarrolla la concepción de justicia basándose en dos principios (Rawls, 2003):

1. Cada persona que participa en una práctica, o que se ve afectada por ella, tiene igual derecho a la más amplia libertad compatible con una similar libertad para todos.

2. Las desigualdades son arbitrarias, a no ser que pueda razonablemente esperarse que redundarán en provecho de todos, y siempre que las posiciones y cargos a los que estén adscritas, o desde los que puedan conseguirse, sean accesibles a todos.

Con estos principios la justicia la expresa en tres ideas: libertad, igualdad y recompensa por servicios que contribuyan al bien común. Es verdad que la justicia es un bien común, sin embargo, expresado en estas ideas es difícil alcanzarlas de manera equitativa.

En esta búsqueda de justicia con equidad descubre que en este proceso surgen las desigualdades y las define como las diferencias en los beneficios y cargas vinculados directa o indirectamente a ellos, tales como el prestigio y riqueza, o sujeción a imposición fiscal y a servicios obligatorios, es decir en la distribución resultante que una práctica establece o hace posible, de las cosas que los hombres se esfuerzan por alcanzar o por evitar (Rawls, 1971).

A pesar de que el individuo se encuentra en esta posición de poder para decidir qué ser, siempre hay que atender reglas que regulan las consecuencias concretas reales o posibles de cada acción individual, es decir, bajo qué circunstancia alcanzamos el bienestar o la felicidad, Sen (2003) señala que en condiciones de desigualdad y de opresión a veces seculares, los oprimidos pueden reducir sus deseos hasta el punto de no poder, osar o querer imaginar una vida mejor y menciona el caso de las mujeres doblemente oprimidas del tercer mundo. 
Por su parte, Rawls (Sen, 2003) dice que no se puede comprar el bienestar al precio de la libertad, pero la eficiencia económica o la prosperidad global tampoco pueden justificar un deterioro de la posición de los menos aventajados, por lo que en realidad lo importante es contar con los siguientes principios:

- Los derechos y libertades cívicas básicas

- La libertad de desplazamiento y la libre elección de ocupación en un marco de diversas oportunidades

- Los poderes y las prerrogativas de los puestos y cargos de responsabilidad en las instituciones políticas y económicas de la sociedad

- Los ingresos monetarios y la riqueza

- Las bases sociales del respeto a sí mismo

Y aunque estos principios son deseables, según Sen (2003) no solucionan el problema de desventaja porque la diversidad social y humana crean amplias variantes (clase, género, educación, condición ecológica o deficiencia física) en lo que la gente es capaz de convertir recursos en libertades reales.

Sen (2003) además explica que lo importante es que las personas disfruten de la libertad de escoger la vida que tiene una buena razón de valorar, lo que se trata de distribuir en modo equitativo no tan sólo ingresos y recursos, sino capacidades para desarrollar funciones humanas fundamentales, es decir, capacidad de vivir una vida digna y sensata más que oportunidad de acumular bienes. Por lo anterior el ingreso es un indicador importante pero relativo del verdadero bienestar, que supone una infraestructura de bienes públicos más complejo que el mecanismo distributivo.

En este enfoque de capacidades, Sen y Casas (1995) se basan en la utilidad, en el que el bienestar de un agente es una función de todas las diversas cosas que éste es capaz o puede hacer: poseer, comer, leer, participar en la actividad política y otras actividades, parte de la idea de que vivir puede considerarse como algo que consiste en un conjunto de funcionalidades interrelacionadas, tales como el estar adecuadamente alimentado, el evitar la mortalidad prematura y el ser feliz como ejemplos de funcionamiento. 
Hay que tener presente que los bienes y valores de los hombres cambian con la evolución de las culturas por lo que es importante considerar la pluralidad de capacidades (Sen 2003).

Esta pluralidad de capacidades humanas debe concretarse no sólo por la voluntad del individuo sino que es necesario un proceso de inversión en el recurso humano, en este sentido se habla entonces de capital humano.

Por su origen el capital humano es innato o adquirido, en el caso del primero comprende aptitudes de tipo físico e intelectual que pueden verse alteradas debido a las condiciones de alimentación y salud. El segundo se irá formando a lo largo de la vida de los sujetos a través de la educación formal e informal y por la experiencia laboral y el sistema de valores de los sujetos, que determinan su rendimiento en el trabajo junto a las aptitudes innatas (Jiménez y Simón 2002).

Por su parte Rawls (2003) considera que quienes hayan sido favorecidos por la naturaleza, son quienes pueden beneficiarse de su buena fortuna sólo en la medida en que mejoren su situación de quienes hayan sido perjudicados. En este sentido la teoría del capital humano es elemental para mejorar el nivel de vida, es decir, quienes tengan dones y motivaciones similares deben tener aproximadamente las mismas oportunidades de alcanzar posiciones de autoridad política, con independencia de su clase económica y social.

Por su parte Becker (Jiménez y Simón 2002) define el capital humano como un stock inmaterial imputable a una empresa, una opción individual y una inversión en algo intangible pero acumulable y utilizable en el futuro.

Según Terrones y Calderón (1993) invertir en educación especialmente en los países de América Latina será sin duda un paso hacia el desarrollo económico y por lo tanto para mejorar el nivel de vida de las personas por varias razones:

a) Aumenta la capacidad productiva del individuo, pues mejora su capacidad de aprendizaje y de acceder a nueva información. 
b) Mejora la capacidad creativa del individuo, generando no sólo innovación técnica sino también innovaciones institucionales.

c) Produce familias más educadas, posibilitando un ambiente familiar y social más propicio para el mejor desarrollo de las futuras generaciones en los planos intelectual, corporal y nutricional.

d) Eleva el costo de oportunidad de tener y mantener hijos, generando así una menor tasa de fertilidad y por ende un menor crecimiento poblacional.

El primer Informe sobre Desarrollo Humano de 1990 creado por el PNUD introdujo una nueva forma de medir el desarrollo mediante la combinación de indicadores de esperanza de vida, logros educacionales e ingreso en un índice de desarrollo humano compuesto: el IDH. Lo innovador del IDH fue la creación de una estadística única que serviría como marco de referencia tanto para el desarrollo social como para el económico. El IDH define un valor mínimo y uno máximo para cada dimensión y luego muestra la posición de cada país con relación a estos valores objetivos, expresados mediante un valor entre o y 1 (PNUD, 15 de julio 2010).

El Informe sobre Desarrollo Humano de 1995 presentó dos nuevas mediciones de desarrollo humano que destacan la situación de las mujeres: el Índice de Desarrollo ajustado por Género (IDG) y el Índice de Potenciación de Género (IPG).

El primer índice, el Índice de Desarrollo ajustado por Género (IDG), mide el progreso con las mismas variables básicas que el IDH, pero refleja las desigualdades en términos de progreso entre mujeres y hombres. La metodología utilizada penaliza la desigualdad, de manera tal que el IDG disminuye cuando descienden los niveles de progreso tanto de mujeres como de hombres a nivel nacional o cuando aumentan las disparidades en el progreso.

La segunda medida, el Índice de Potenciación de Género (IPG), es una medida de acción. Evalúa el progreso de las mujeres en su participación en foros políticos y económicos. Analiza hasta qué punto las mujeres y los hombres tienen la posibilidad de participar activamente en la vida política 
y económica y en la toma de decisiones. Mientras que el IDG se concentra en la ampliación de las capacidades, el IPG se refiere al uso de esas capacidades para aprovechar las oportunidades de la vida.

Las dos medidas se han utilizado como herramientas de difusión y de supervisión para el análisis del desarrollo humano y los debates sobre políticas relacionados con el género.

En el IDH 2009 se aplicaron las metodologías del IDG y del IPG a nivel mundial para calificar a 155 países por IDG y a 109 países por IPG. También es posible aplicar las mismas metodologías en el ámbito nacional y en el subnacional, mediante IDG e IPG desagregados. Así, es posible comparar diferencias entre regiones, grupos étnicos, etcétera (PNUD 2009).

El IDG es el segundo indicador que se calculó en el estudio, el cual toma la desigualdad de logros entre mujeres y hombres.

Este índice ajusta el promedio de logro para reflejar las desigualdades entre hombres y mujeres en las siguientes dimensiones:

- Una vida larga y saludable, expectativa al nacer.

- Conocimiento, medido por la tasa de alfabetización de adultos y la tasa bruta combinada matriculada en escuelas primarias, secundarias y medio superior.

- Un nivel de vida digno, medido por los ingresos percibidos estimados (dólares PPC).

El estudio conjunto de indicadores económicos con enfoque de género lo define según Careaga, Barquet, y Brenes (2004) como "el conjunto de ideas, creencias y atribuciones sociales, construidas en cada cultura y momento histórico, tomando como base la diferencia sexual; a partir de ello se construyen los conceptos de masculinidad y feminidad, los cuales determinan el comportamiento, las funciones, oportunidades, valoración y las relaciones entre hombres y mujeres", permite entender su aplicación al proceso de desarrollo, el análisis de género mide cuanto influyen los programas y políticas en las mujeres y en los hombres de manera diferencial. 
La desigualdad del género ha llevado a otros estudiosos, como Gary Becker, a estudiar el tema de la mujer dentro del análisis de la economía neoclásica moderna en sus trabajos desarrollados sobre las diferencias salariales a mediados de 1950 en su libro titulado La economía de la discriminación, simultáneamente con el incremento de los movimientos sociales sobre la mujer moderna (Albelda, 1997).

En el enfoque de género según el principio ricardiano de la ventaja comparativa, Becker (Vara, 2006) menciona que en la mayoría de las parejas el varón casado tendría a especializarse en el trabajo de mercado, al tiempo que la esposa mostraría una especialización relativa en la producción doméstica que incluye el cuidado de los hijos. Al respecto afirman que en esta situación de incertidumbre y aversión al riesgo, los empresarios pueden utilizar criterios de discriminación estadístico por sexo en la contratación y promoción de sus empleados, asociando matrimonios con hijos con estabilidad laboral, en el caso de los varones y mayor probabilidad de ausentismo y abandono voluntario en el caso de las mujeres.

Una sociedad más justa y con igualdad social requiere de nuevos indicadores que midan no sólo la parte económica sino también lo humano en el sentido más amplio, es decir, que considere los valores y las aspiraciones de las personas para el acceso a una vida prolongada y saludable, con los satisfactores que le permitan desarrollarse productivamente y de acuerdo con sus intereses.

La desigualdad de género es menor cuando se logra un mayor desarrollo y éste es difícil de alcanzar cuando las oportunidades de educación, salud e ingreso no son suficientes para concientizar a la sociedad de la importancia de su participación para diseñar y reorientar las políticas públicas. $\mathrm{Al}$ respecto, según Canto (Otoño 2008) la falta de desarrollo es un factor de ineficiencia de las políticas, para hacerle frente a esta situación, la participación democrática es un componente necesario, se requiere también romper un círculo vicioso que podría describirse de la siguiente manera:

No alcanzamos el desarrollo porque no tenemos una adecuada participación en las decisiones públicas y no hay una demanda participativa en la sociedad porque no contamos con el nivel de desarrollo necesario. 
En la estructura de una sociedad hay tres importantes factores que influyen en la igualdad de género (Banco Mundial y Universidad de Oxford, 2001):

- Instituciones, incluyendo normas sociales, leyes y mercados.

- El hogar.

- La economía.

El Estado puede intervenir de muchas maneras. Puede gravar y subsidiar, persuadir y regular, prohibir y sancionar, y proveer de servicios públicos. Puede financiar (o subsidiar) las inversiones para contrarrestar las desigualdades de género.

Una de las áreas más difíciles para la intervención del Estado es el hogar, donde las desigualdades se derivan en parte de las diferencias biológicas, de las normas tradicionales sobre los roles de género y de desigualdad de poder de negociación entre mujeres y hombres.

En cuanto a lo económico es importante destacar que la división del trabajo en el hogar diferencia las capacidades del hombre y la mujer para desempeñar una actividad económica que le proporcione un ingreso remunerado y por lo tanto un mayor nivel de bienestar.

Los roles se suelen identificar con las etiquetas que se utilizan para designar distintas posiciones sociales en una cultura, los cuales por lo general se diferencian según la edad, el sexo, la relación de parentesco, la ocupación o el status social (Bronfenbrenner, U., 1987).

Es claro que las políticas públicas tienen un papel central en la transformación de los estereotipos que surgen en la sociedad debido a la crisis, especialmente derivados de la pobreza, en este sentido estas políticas se orientan a promover la reflexión sobre las transformaciones en los papeles y las relaciones de género que ellas mismas inducen, así como apoyar los procesos de cambios, previendo las situaciones de violencia intrafamiliar que éstos pudieran acelerar y que pudieran orientarse hacia la democratización de las relaciones familiares, entre los géneros y entre las generaciones (Schmukler, 2000). 
El estudio de las oportunidades, funcionamientos y capacidades de las personas para mejorar su nivel de vida es complicado, ya que la falta de información estadística limita la utilización de las variables para su medición y con ello el diseño de las políticas públicas que consideren el bienestar público.

\section{METODOLOGÍA}

Para la construcción de los indicadores de desarrollo humano por razones de disponibilidad y con el fin de corroborar la teoría, se anexan variables que se aproximan a las aplicadas en la estimación aplicada por PNUD. Para ello se requirió de la obtención de datos a partir de la aplicación de cuestionarios a una muestra representativa de la población y se complementó con información estadística del Consejo Estatal de Población del estado de Campeche (COESPO), del Instituto Nacional de Estadística e Informática (INEGI) y datos estandarizados del PNUD, en virtud de que se tuvo que adaptar la información requerida en la metodología para obtención de los índices a nivel municipal para su construcción a nivel de una sección municipal.

La información se analizó considerando la interacción a partir de las estrategias de género que desarrollan los miembros de los grupos domésticos en la zona, en relación con su acceso a la tierra y a los programas de apoyo al campo y a la pesca, con las políticas de empleo, el acceso a la salud y a la educación. En esos ámbitos los miembros de los grupos domésticos entran en relación con los programas, las normatividades y las prácticas y visiones de los técnicos y funcionarios encargados de dichas políticas.

\subsection{Descripción del área de estudio}

El proyecto se desarrolló en los poblados y ejidos de la sección municipal de Sabancuy, incluyendo a la propia cabecera urbana. La sección pertenece al sur occidental del municipio El Carmen y está integrado por 12 ejidos: General Abelardo L. Rodríguez, General Ignacio Gutiérrez, José María Pino Suárez, Chicbul, Independencia, Checubul, Cristalina, Nicolás Bravo, Sabancuy, Oxcabal, Plan de Ayala y Enrique Rodríguez Cano; 
con el siguiente estatus administrativo: 11 localidades rurales que cuentan con menos de 1000 habitantes, y una localidad urbana (Sabancuy) con una población superior a los 3000 habitantes, asimismo, se encuentran 30 pequeñas rancherías dispersas con escasa población. La sección está ubicada a orillas del nororiental estero de la Laguna de Términos, a dos kilómetros del Golfo de México.

El área de estudio destaca por ubicarse en el segundo municipio en importancia en el estado de Campeche no sólo por la explotación del petróleo de la Sonda de Campeche sino por las magníficas condiciones portuarias y zonas de pesca, en la cual destaca la captura de camarón, lo cual supondría que se presentan más oportunidades en cuanto al empleo, el acceso a la educación y la salud.

En las comunidades de la sección de Sabancuy, menos de la mitad de la población se encuentra en el PEA, una constante que se refleja en todas las comunidades, lo que indica que no hay muchas fuentes de empleo en esta región. En lo que respecta al comportamiento del salario mínimo, encontramos que la población económicamente activa que se encuentra en cada uno de los diferentes niveles de salario mínimo, destaca que las localidades de Sabancuy, Chicbul, Checubul, Ignacio Gutiérrez, Oxcabal, la Cristalina e Independencia perciben entre uno y dos salarios mínimos y las poblaciones Abelardo Rodríguez, Enrique Rodríguez Cano, Plan de Ayala, Nicolás Bravo y José María Pino Suárez se percibe un salario mínimo en promedio (INEGI, 2000).

En materia de educación la escolaridad en las localidades se encuentra en menos de seis años, y el grado promedio de educación es de 5 años. También pudimos constatar que en la gran mayoría existen más hombres que mujeres, incluso hasta en localidades de mayor número como en Sabancuy (INEGI, 2005).

\subsection{Diseño de la muestra}

Para el levantamiento de las encuestas fue necesario calcular una muestra representativa, para ello se tomó como fuente la población de viviendas; según el conteo de población y vivienda 2005 del Instituto 
Nacional de Estadística Geografía e Informática (INEGI), la muestra está compuesta por 3,402 viviendas que se distribuyeron en 12 localidades de la sección municipal de Sabancuy. El tamaño de la muestra se obtuvo considerando que cuando se estima el valor de una proporción y el muestreo se va a hacer sin reemplazo en una población finita se emplea la siguiente fórmula (Wayne, 1981):

$$
n=\frac{z^{2} N p q}{z^{2} p q+(N-1) d^{2}}
$$

Donde:

$\mathrm{d}=$ error permitido

$Z=1.96$ que es el valor de la distribución "Z" para una confiabilidad del $95 \%$

$\mathrm{P}=\mathrm{p}=\%$ estimado

$\mathrm{q}=100-\mathrm{p}$

$\mathrm{N}=$ tamaño de la población

$\mathrm{n}=$ tamaño de la muestra

Tabla 2.- Porcentaje de la muestra por localidad

\begin{tabular}{|l|c|c|c|}
\hline Localidades & Total de viviendas & Representa & Porcentaje en la muestra \\
\hline Abelardo L. Rodríguez & 236 & $6.94 \%$ & 24.002 \\
\hline Ignacio Gutiérrez & 136 & $4.00 \%$ & 13.832 \\
\hline José María Pino Suárez & 75 & $2.20 \%$ & 7.628 \\
\hline Plan de Ayala & 170 & $5.00 \%$ & 17.290 \\
\hline Chicbul & 378 & $11.11 \%$ & 38.444 \\
\hline Independencia & 65 & $1.91 \%$ & 6.611 \\
\hline Checubul & 383 & $11.26 \%$ & 38.953 \\
\hline La Cristalina & 156 & $4.59 \%$ & 15.866 \\
\hline Nicolás Bravo & 75 & $2.20 \%$ & 7.628 \\
\hline Sabancuy & 1494 & $43.92 \%$ & 151.947 \\
\hline Oxcabal & 133 & $3.91 \%$ & 13.527 \\
\hline Enrique Rodríguez Cano & 101 & $2.97 \%$ & 10.272 \\
\hline Total & & & $\mathrm{n}=346$ \\
\hline
\end{tabular}


Notamos en la tabla, que con la fórmula aplicada (muestra en proporciones finitas) obtuvimos una muestra total de 346 y se distribuyó según lo que representa cada localidad en el total de la población de viviendas.

\subsection{Obtención de índices de desarrollo}

Lograr una elección de vida con libertad sin duda requiere de una serie de factores y condiciones que puedan proporcionar el nivel de bienestar social alcanzable, es por ello que en el presente estudio nos referiremos específicamente a dos indicadores de desarrollo (Índice de Desarrollo Humano e Índice de Desarrollo Relativo al Género), los cuales nos explicarán cuál es el grado de desarrollo en la sección municipal de Sabancuy, El Carmen, Campeche, por lo que es importante hacer mención de la definición, el procedimiento de su obtención y la interpretación de sus resultados.

\subsubsection{IDH}

Para el cálculo del componente de salud del IDH, se consideró como valor efectivo la esperanza de vida al nacer a nivel estatal para el año 2008, por no encontrarse información desglosada a nivel municipal y como valor máximo y mínimo la media obtenida de los datos históricos del periodo de 1990-2008, con el fin de obtener un resultado más aproximado a la realidad local.

Aplicando la fórmula del PNUD tenemos:

$$
\text { Índice de salud }=\frac{75.06-73.5}{76.1-73.5}=0.6000
$$

Para el caso del índice de educación, se tomó como datos la tasa de alfabetización de adultos, la cual fue de $88.34 \%$ (población de 15 años o más), también se utilizó la tasa de asistencia escolar de la muestra (población de 6 a 24 años de edad), que fue de 56.12\%. Como valor máximo y mínimo se utilizó el parámetro establecido por el Programa de las Naciones Unidas para el Desarrollo (PNUD) que es de 100\% y o\%, respectivamente. $\mathrm{Al}$ aplicar la fórmula se obtuvo lo siguiente: 
TASA DE ALFABETIZACIÓN DE ADULTOS $=\frac{88.34-0}{100-0}=0.8834$

TASA DE ASISTENCIA ESCOLAR $=\frac{56.12-0}{100-0}=0.5612$

Por lo tanto, el índice de educación es:

ÍNDICE DE EDUCACIÓN $\left.=\frac{2}{3}(0.8834)\right)+\frac{1}{3}(0.5612)=0.7759$

En el componente de ingreso se tomó como base el ingreso promedio mensual de la Población Económicamente Activa de los resultados de las encuestas, para obtener el ingreso promedio per cápita anual de la PEA ajustado mediante el factor de conversión a términos de Paridad de Poder de Compra en dólares estadunidenses (dólares PPC) tomando el valor de 14.64 pesos por dólar. Los valores máximos y mínimos de referencia, son los establecidos oficialmente por la PNUD de 40,000 y 100 dólares PPC, respectivamente. $\mathrm{Al}$ aplicar la fórmula se obtuvo:

ÍNDICE DE INGRESO $=\frac{\log \square(2420.58)-\log \sum(100 \Sigma)}{\log \square(400,000)-\log \sum(100 \Sigma)}=0.5318$

Una vez obtenidos los tres parámetros se calculó el IDH:

$\mathrm{IDH}=\frac{1}{3}(0.6)+\frac{1}{3}(0.7759)+\frac{1}{3}(0.5318)=0.6358$

Para su interpretación, el IDH hace uso de un valor, el cual varía entre o y 1, e indica la distancia que tiene que recorrer un país o región para lograr el valor máximo posible de desarrollo, que es igual a 1.

Lopez et al. (2006) mencionan que los parámetros utilizados por la PNUD para clasificar a los países es el siguiente: 
- Los países con índices entre o.8 y 1, se encuentran en un desarrollo humano alto.

- Países con un índice entre 0.5 y 0.799 es un desarrollo humano medio.

- Los países cuyo índice es menor a 0.499 presentan un desarrollo humano bajo.

De acuerdo con los criterios anteriores y el resultado obtenido en el IDH de 0.6358 , la sección municipal de Sabancuy tiene un desarrollo humano medio, por lo que aún queda camino por recorrer para avanzar hacia el progreso ideal. Si comparamos con el IDH del municipio de El Carmen al que pertenece la sección de Sabancuy, de 2006 a 2007 que fue de 0.807 (CEIDAS, 2008) representa un desarrollo humano alto, la diferencia radica en que los datos a nivel municipal son afectados por la actividad económica (petróleo), pero de poca igualdad en cuanto a los beneficios económicos y sociales.

Este indicador incorpora información asociada con las características de las personas y su calidad de vida, lo que lo hace una medida socioeconómica de desarrollo muy interesante porque puede servir como referencia en el diseño de las políticas públicas que repercutan en el nivel de vida de las comunidades que integran la sección municipal de Sabancuy.

\subsubsection{IDG}

Para la obtención del IDG se utiliza un procedimiento similar al IDH, es decir, se calculan los indicadores de salud, ingreso y educación, pero el resultado de IDG hace precisión en las desigualdades de género en las capacidades básicas, pero desagregado por género, buscando mayor sensibilidad a la equidad en la condición de los sexos, especificando los máximos y mínimos (límites para cada uno de los indicadores) de los componentes. Las fórmulas aplicadas para su obtención son las siguientes:

\section{Componente Salud}

Para este componente se consideró como valor efectivo la esperanza de vida al nacer, para ambos sexos, a nivel estatal, por no encontrar referencia desglosada a nivel municipal y como máximo y mínimo la esperanza 
de vida al nacer, de los datos históricos en el periodo 1990-2008, con el fin de obtener un resultado más aproximado a la realidad local, obteniéndose los siguientes resultados:

Índice esperanza de vida mujeres $=\frac{77.38-75.80}{78.50-75.80}=0.5852$

Índice de esperanza de vida hombres $=\frac{72.74-71.2}{74-71.2}=0.5500$

Índice de salud igualmente distribuido:

$$
\operatorname{IIDS}=\left\{\left[0.4935\left(0.5852^{-1}\right)\right]+\left[\left[0.5064\left(0.5500^{-1}\right)\right]\right\}^{-1}=0.5668\right.
$$

La proporción hombres y mujeres de la población es tomada de la muestra en la cual 50.64\% son mujeres y $49.35 \%$ hombres.

\section{COMPONENTE EDUCACIÓN}

Para el cálculo del índice de educación se utilizó la tasa de alfabetización de adultos para el hombre y la mujer (población de más de 15 años) y la tasa de asistencia escolar (población de 6 a 24 años de edad), ésta se obtuvo de la población de hombres y mujeres alfabetizados y asistencia escolar de la muestra que fue de 0.9216 y 0.843 y 0.5611 y 0.5612, respectivamente.

$$
\text { Tasa de alfabetización mujeres }=\frac{2}{3}(0.843)+\frac{1}{3}(0.5612)=0.7490
$$$$
\text { Tasa de alfabetización hombres }=\frac{2}{3}\left(0.9216+\frac{1}{3}(0.5611)=0.8014\right.
$$

$$
\text { IIDI }=\left\{\left[0.4935\left(0.7490^{-1}\right)\right]+\left[\Sigma 0.5064\left(0.8014 \Sigma^{-1}\right)\right]\right\}^{-1}=0.7747
$$




\section{COMPONENTE INGRESO}

Para el cálculo del índice del ingreso se tomó como valor efectivo el ingreso promedio per cápita de la población económicamente activa de la muestra de hombres y mujeres (también ajustado mediante el factor de conversión a términos de dólares PPC con una paridad de 14.64 pesos por dólar) y fue de $2,552.87$ y $1,943.86$ respectivamente. Los valores máximos y mínimos de referencia son los establecidos oficialmente por la PNUD de 40, 000 y 100 dólares PPC, respectivamente.

$$
\begin{aligned}
& \text { Índice de ingreso mujeres }=\frac{\log \square(1,943.86)-\log \Sigma(100 \Sigma)}{\log \square(40,000)-\log \Sigma(100 \Sigma)}=0.4952 \\
& \text { Índice de ingreso hombres }=\frac{\log _{\square}(2,552.87)-\log \Sigma(100 \Sigma)}{\log _{\square}(40,000)-\log \Sigma(100 \Sigma)}=0.5407
\end{aligned}
$$

$$
\mathrm{IIDI}=\{[0.4935(0.4952-1)]+[0.5064(0.5407-1)]\}-1=0.5173
$$

El desempeño de cada componente se expresa como un valor entre o y 1 y finalmente se suma cada componente para obtener el IDG, mediante la siguiente fórmula:

$$
\mathrm{IDG}=\frac{1}{3}(0.5668)+\frac{1}{3}(0.7747)+\frac{1}{3}(0.5173)=0.6195
$$

\section{ANÁLISIS DE RESULTADOS}

Los resultados obtenidos por componente de los indicadores de desarrollo en la Sección Municipal de Sabancuy muestran que el componente de educación se encuentra en la primera posición, lo que indica que para las personas el acceso al conocimiento es de gran importancia ya que la ven como una oportunidad de desarrollo y de bienestar social, en este sentido las políticas públicas se están orientando a atender la necesidad de educación mediante la infraestructura educativa existente; encontramos 
que para el ciclo 2005-2006, había 15 escuelas primarias, donde asistieron 2,382 alumnos inscritos, de los cuales 1,228 son mujeres y 1,154 hombres. A nivel secundaria cuenta con 4 escuelas y 2 telesecundarias con 497 mujeres y 473 hombres, esto es un total de 970 alumnos, además existen 2 escuelas preparatorias con 436 alumnas (sólo mujeres) localizadas en Chicbul y Sabancuy (COESPO, 2006).

Como se puede observar, la educación que se imparte en la sección es muy básica y la desigualdad entre hombres y mujeres es muy relativa, actualmente la brecha educativa persiste porque los accesos a los diferentes niveles de educación se han restringido para ambos sexos por diferentes factores, encontrando entre los principales el ingreso, la distancia de las escuelas y las costumbres que obligan a las mujeres a convertirse en madres desde muy temprana edad y dedicarse a su familia. Sin embargo, las que han logrado alcanzar el nivel bachillerato, no solamente se dedican al hogar sino que realizan otras actividades informales (trabajadoras domésticas, realizan trabajos de belleza o venden productos por catálogo o en la calle) para contribuir con el gasto familiar.

Gráfica 1. Componentes del IDH

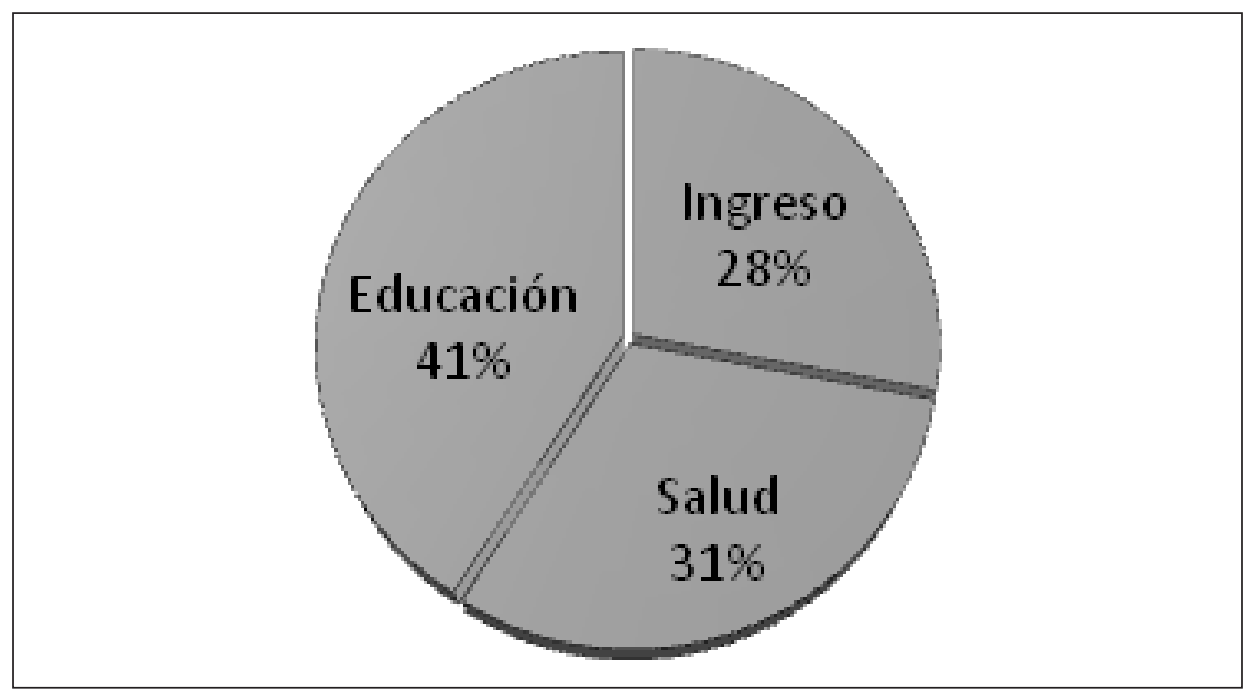

Fuente: Elaboración propia con los resultados obtenidos en 2008. 
En relación con el componente de salud, el cual ocupa el segundo lugar, de acuerdo con los resultados obtenidos, la población recibe una atención médica proporcionada generalmente por los centros de salud, esto es, 93.7\% utiliza el servicio público que existe en las comunidades y $6.3 \%$ es atendida por el servicio privado.

Es importante mencionar que los centros de salud atienden a la población en general (33.1\%) y también a las personas que cuentan con algún programa social como es oportunidades y seguro popular, en este sentido es claro que estas unidades médicas no son suficientes y en muchos casos no cuentan con el personal capacitado (en ocasiones son enfermeras o pasantes de medicina) ni con la infraestructura adecuada y por lo tanto atienden enfermedades que no son de gravedad. Y aunque algunas personas tienen acceso a instituciones especializadas en cuanto al servicio como es el IMSS (12.9\%) e ISSSTE (3.2\%), esta es una proporción no significativa, por lo que la reflexión en este rubro para el diseño de las políticas en materia de salud se debe centrar en la cobertura y la calidad de atención de la salud a la población para un desarrollo de vida sana.

El componente que ocupa el último lugar es el índice del ingreso, el cual presenta una diferencia significativa respecto a los de educación y salud. Las oportunidades de trabajo en la sección han disminuido debido al agotamiento de los recursos naturales, a la vocación de los pobladores y a la migración de zonas rurales a las urbanas y al extranjero que han incidido especialmente en las actividades del campo, y aunque ésta sigue siendo el sector que sustenta la actividad económica, su crecimiento es muy lento, lo que se refleja en los bajos ingresos que perciben los trabajadores de las comunidades de la sección de Sabancuy.

Se obtuvo también que de la población económicamente activa, 80.7\% de las personas que trabajan son hombres y $19.3 \%$ son mujeres. En relación con los hombres $77.27 \%$ se dedica principalmente al sector primario (agricultura y pesca), 15.24\% a actividades terciarias y sólo $7.49 \%$ a actividades del sector secundario (construcción y Pemex). 
En el caso de las mujeres la situación es diferente, $76.40 \%$ se dedica al sector terciario (principalmente al comercio), 21.34\% al sector primario y tan sólo $2.24 \%$ al sector secundario.

En lo que respecta a las principales posiciones en el trabajo del padre de familia se obtuvo que $38.5 \%$ es empleado y $27.1 \%$ es jornalero, con respecto a la madre de familia $44.4 \%$ tiene un negocio propio y $43.2 \%$ es empleada.

\section{Gráfica 2. Componentes del IDG}

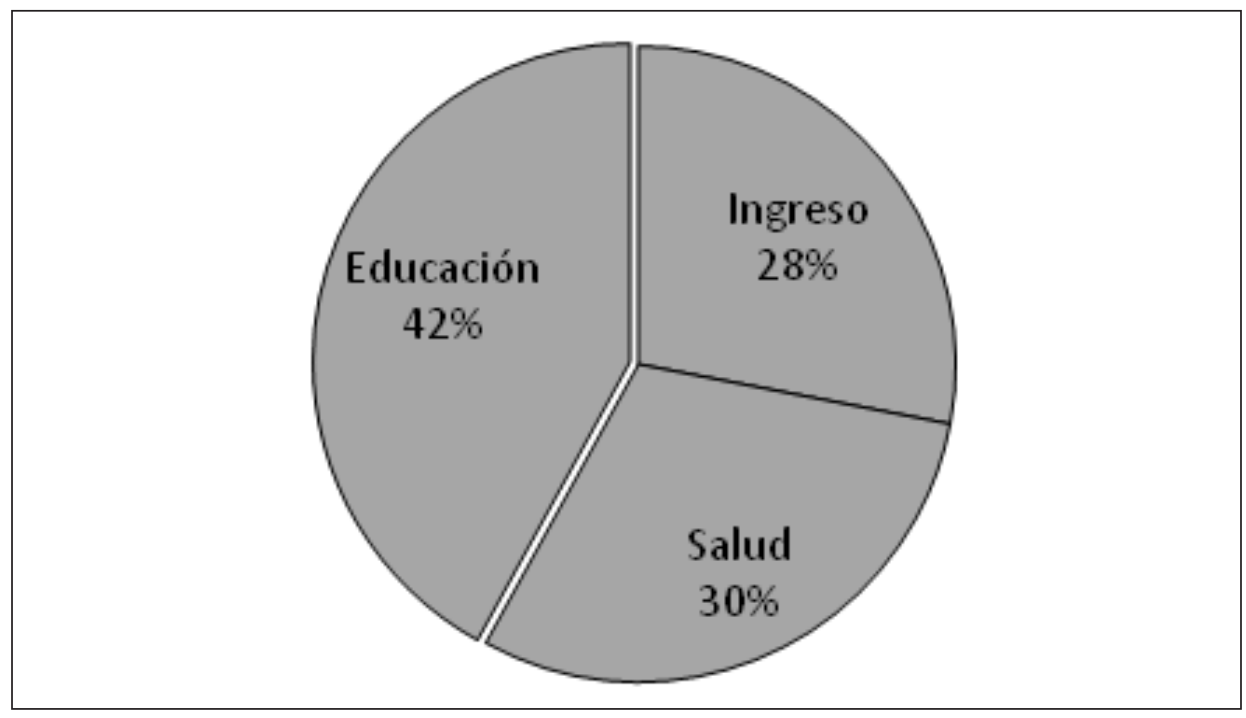

Fuente: Elaboración propia con los resultados obtenidos en 2008.

Lo anterior explica por qué el componente de ingreso es más bajo en ambos índices (IDH e IDG) que el de educación y salud, debido a que las ocupaciones laborales de los hombres y las mujeres siguen siendo definidas por los roles que socialmente juegan, y aunque es cierto que la mujer y el hombre actualmente están incorporándose a actividades que tradicionalmente no corresponden a su género, la desigualdad especialmente para las mujeres es notoria y evidente. 
En relación con el IDG, el componente de educación subió un punto porcentual, con respecto de lo obtenido en el IDH, debido a que el acceso a la educación se ha ampliado y a las mujeres se les permite estudiar. Pero el componente de salud en este caso perdió un punto con respecto del IDH.

\section{CONCLUSIONES}

El IDH obtenido en la sección municipal de Sabancuy, Carmen, Campeche, fue de 0.6358 (desarrollo medio) y el IDG fue ligeramente menor al resultar con un 0.6195 , que también se considera dentro de los parámetros de un desarrollo medio, lo que concuerda con las teorías de que si se toma en cuenta la desigualdad de género, el índice de desarrollo disminuye.

En el IDH 31\% corresponde al índice de salud, 41\% al índice de educación y $28 \%$ al índice de ingreso. El más bajo fue el índice de ingreso, esto se corroboró con la proporción de salarios mínimos obtenida. El IDG lo determinan en 30\% el índice de salud, 42\% el índice de educación, y el ingreso es el más bajo con $28 \%$, esto último coincide con el IDH, debido a que ese componente continúa siendo el principal factor de disminución del desarrollo. El IDG es un indicador útil para reflejar la desigualdad promedio en logros por género en las mismas dimensiones que analiza el IDH, esto nos permite concluir que hay una pérdida de desarrollo en esta sección municipal al considerar el género.

Por ello se debe hacer énfasis en mejorar la preparación académica de la población, para generar mayores oportunidades de empleo y así puedan tener acceso a mejores niveles de vida, lo anterior aunado a un gobierno incluyente y eficiente, que proporcione además el financiamiento económico necesario para reactivar las actividades productivas estratégicas de estas localidades con vocación de género.

El escenario de la sección municipal de Sabancuy se caracteriza por un crecimiento lento, las políticas públicas no han impactado en el progreso de los ejidos y comunidades y por lo tanto las actividades 
económicas tradicionales de esta región han venido en detrimento en los últimos veinte años, específicamente la agricultura y la pesca, esta última afectada considerablemente por el establecimiento de las plataformas de Pemex que afectan el entorno natural y además les limita el área para pescar, repercutiendo en la disminución de los ingresos suficientes que les permita tener un mejor nivel de vida. Es por ello que la obtención de indicadores socioeconómicos como el IDH y el IDG deben servir como instrumentos y orientadores para el diseño de las políticas públicas que consideren las vocaciones productivas de las personas con un enfoque de género, así como programas de salud y de educación cuyos objetivos sea la formación de capital humano que desarrolle sus capacidades físicas e intelectuales para mejorar el bienestar de la sociedad. 


\section{BIBLIOGRAFÍA}

Albelda, R. (1997) Economics and Feminism Disturbances in the Field. Estados Unidos: Prentice Hall International.

Banco Mundial y Universidad de Oxford. (2001) Engendering Development through Gender Equality in Rights, Resources and Voices. A word Bank Policy Research ReportOxford: Copublicación del Banco Mundial y Universidad de Oxford.

Bronfenbrenner, U. (1987) La ecología del desarrollo humano, España, Paidós.

Canto, M. (2008) "Gobernanza y participación ciudadana en las políticas púbicas frente al reto del desarrollo". Política y cultura, 30. México.

Careaga, G. Barquet, M. y Brenes, I. (2004) El ABC del género en la administración pública, México: Instituto Nacional de las Mujeres.

CEIDAS (11 de junio del 2008) Información del estado de Campeche, (en línea) <www.ceidas.org/at_map_campeche_esp.shtml.> (Consultado 12 de enero de 2009).

COESPO (2006) Informes estadísticos. Campeche.

Hernández, D. y Székely, M. Capítulo 3. Medición del bienestar en México en los inicios del siglo XXI. En Székely, M. (2005) Números que mueven al mundo: La medición de la pobreza en México. Miguel Ángel Porrúa. México.

INEGI (2000) Censo de Población y Vivienda 2000. Instituto Nacional de Estadística Geografía e Informática. México.

INEGI (2005) Conteo de Población y Vivienda 2005. Instituto Nacional de Estadística Geografía e Informática. México. 
Jiménez G. y Simón B. (2002) Una nueva perspectiva en la medición del capital humano. Unirioja. España.

Jiménez, L. (2001) Desarrollo sostenible y economía ecológica. Editorial Síntesis, España.

López, L., Rodríguez, E. y Vélez, R. (2006) Estimación de IDH estatal en México, análisis de sensibilidad a distintas decisiones metodológicas y comparaciones internacionales, (en línea) <www.sedesol.gob.mx/archivo/80/588/file/docu_8_2006> (Consultado el 28 de febrero de 2009).

PNUD (2009) Informe sobre desarrollo humano 2009. Grupo Mundi Prensa. Estados Unidos.

PNUD. Antecedentes. Informes sobre desarrollo humano (En línea) <http://hdr.undp.org/es/desarrollohumano/idh/ > (Consultado el 15 de julio de 2010).

PNUD. Medición de la desigualdad: el Índice de Desarrollo ajustado por Género (IDG) y el Índice de Potenciación de Género (IPG). (En Línea)<http://hdr.undp.org/es/estadisticas/indices/idg_ipg/> (Consultado el 15 de julio de 2010).

Rawls, J. (1971) Teoría de la justicia. Fondo de Cultura Económica. México.

Rawls, J. (2003) "Justicia como equidad" Revista Española de Control Externo. No. 13 Vol. 5. Dialnet. España. pp. 129-158.

Schmukler, B. (2000) "Orientaciones de género en las políticas sociales en México y en América Latina”. En Cordera, R.y Ziccardi, A. (Eds.) Las políticas sociales de México al fin del milenio. Descentralización, diseño y gestión. Coordinación de Humanidades. Facultad de Economía. Instituto de Investigaciones Sociales. UNAM. 
Sen, A. y Casas J. (1995) Nueva economía del bienestar. Colección Honoris Causa. Universidad de Valencia. España.

Sen, A. (2001). El nivel de vida. Editorial Complutense. España.

Sen, A. (2003) La libertad individual como compromiso social. ILDIS/ Plural Editores. Bolivia.

Terrones, M. y Calderón C. (1993) Educación, capital humano y crecimiento económico: El caso de América Latina. (En línea) <http:// www.grade.org.pe/download/pubs/NPD/NPDo9-2.pdf> (Consultado el 9 de julio de 2010).

Vara, M. (2006) Estudios sobre género y economía. Ediciones AKAL. Madrid, España.

Wayne, D. (1981) Estadística con aplicaciones a las ciencias sociales y la educación. México: Mc Graw Hill.

Zendejas, S. (2003) Política social y formación del estado. Procesos históricos de formación de espacios y sujetos sociales en un municipio rural mexicano. Tesis doctoral. 